\title{
Advanced multi-plane phase retrieval using Graphic Processing Unit: augmented Lagrangian technique with sparse regularization
}

\author{
Artem Migukin*, Vladimir Katkovnik, Jaakko Astola \\ Department of Signal Processing, Tampere University of Technology, \\ Korkeakoulunkatu 10, P.O. Box 527, 33101, Tampere, Finland
}

\begin{abstract}
In our work we demonstrate a computational method of phase retrieval realized for various propagation models. The effects, arising due to the wave field propagation in an optical setup, lead to significant distortions in measurements; therefore the reconstructed wave fields are noisy and corrupted by different artifacts (e.g. blurring, "waves" on boards, etc.). These disturbances are hard to be specified, but could be suppressed by filtering. The contribution of this paper concerns application of an adaptive sparse approximation of the object phase and amplitude in order to improve imaging. This work is considered as a further development and improvement of the variational phase-retrieval algorithm originated in '. It is shown that the sparse regularization enables a better reconstruction quality and substantial enhancement of imaging. Moreover, it is demonstrated that an essential acceleration of the algorithm can be obtained by a commodity graphic processing unit, what is crucial for processing of large images.
\end{abstract}

Keywords: phase retrieval, prior information, constrained optimization, sparse approximation, parallel processing.

\section{INTRODUCTION}

The conventional sensors detect only the intensity of the light, and the phase is systematically lost in measurements. The phase information can recover the shape of the object, making it useful in metrology and imaging, e.g. microscopy, astronomy, material analysis, etc. Since optical phase cannot be measured directly, computational phase recovering techniques are required for imaging and data processing. The traditional computational methods of the wave field reconstruction can be divided into two groups according to the physical implementation: interferometric techniques with a reference beam and phase-retrieval methods with no reference beam. The latter approach is more reliable, robust to various disturbances (as vibrations) and technically simpler.

An efficient iterative algorithm for phase retrieval is originally proposed by Gerchberg and Saxton ${ }^{2}$, initially for a single measurement plane. The common idea consists in iterative replacement of the estimated magnitudes at the observation planes by measured or a priori information ${ }^{3-4}$. It is shown that such iterative phase recovering can be realized either with respect to the object plane ${ }^{5}$ or to measurement planes ${ }^{6}$. Moreover, similar methods are proposed for Fresnel instead of Fourier transforms as the transfer functions of the wave field propagation both for the wave field reconstruction ${ }^{7-8}$ and design ${ }^{9-10}$. In 1982 Fienup systematized the earlier works and introduced some, for now classical, types of phaseretrieval algorithms ${ }^{11}$. Despite the fact that solutions are in general not unique ${ }^{12-13}$, the ambiguity of the reconstructed phase can be substantially reduced using a number of observations ${ }^{14}$, both for the reconstruction ${ }^{15-16}$ and for synthesis ${ }^{17}$. It is recognized that larger number of measurements leads to better reconstruction quality ${ }^{18}$.

Another phase recovering method, proposed by Teague, is phase diversity. The phase is reconstructed by measuring the axial intensity derivative, where the direct solution can be found from two ${ }^{19}$ or multiple defocusing images ${ }^{20}$ with the transport of intensity equation (TIE). TIE based techniques are shown to be computationally efficient, but very noisesensitive $^{21}$ and fail in case of large amounts of noise. Moreover, the result of phase retrieval is object-dependent what means that the measurement setup parameters are hard to be optimized. This suggests application of the iterative algorithm with an adaptive regularization.

\footnotetext{
*artem.migukin@tut.fi; phone: +358401981310; fax:+358331154989

This work was supported by the Academy of Finland project no. 138207, 2011-2014. The postgraduate work of Artem Migukin is funded by Tampere Doctoral Programme in Information Science and Engineering (TISE).
} 
In our work we consider the optimal wave field reconstruction, developed in terms of a variational constrained maximum likelihood formulation as it is originated in ${ }^{1}$. It is found that additional filtering of the reconstructing object distribution results in essential improvement in imaging ${ }^{22}$. For an enhancement of imaging we improve our model of phase retrieval and use the sparse regularization as a modern and one of the most efficient numerical techniques used for this purpose ${ }^{23}$. According to the sparsity hypothesis an approximation of the object amplitude and phase is performed separately with small number of items of very specific basis functions named BM3D-frames ${ }^{24}$. In this paper we analyze the advantage which can be obtained by the incorporation of this sparse approximation (originated in ${ }^{25}$ and developed in ${ }^{26-27}$ ) for various propagation models: free space propagation and plane-to-plane propagation in a $4 \mathrm{f}$ system. Especially for fast processing of large images (say, $1024 \times 1024$ and more) our parallel algorithm is realized with a graphic processing unit (GPU) providing an essential decrease of the algorithm runtime. The results are presented for a commodity GPU using MATLAB: the implementation is easy in use, and it gives an opportunity for further performance improvements.

\subsection{Propagation model}

In our work we consider a typical optical setup, where a coherent complex-valued wave field is assumed to be propagated from an object plane to a parallel measurement plane. This free space forward wave field propagation is traditionally modeled according to the scalar diffraction theory. Let $u_{0}(x)$ and $u_{r}(x), x \in \mathbb{R}^{2}$ denote the wave field distributions at the object and the $r$-th measurement plane, respectively. Assume that $z_{r}$ is a distance between the object and the $r$-th observation plane, $z_{r}=z_{1}+(r-1) \cdot \Delta_{z}, r=1, \ldots \mathrm{K}$, where $\mathrm{z}_{1}$ is the distance from the object to the first measurement plane, $\Delta_{z}$ is the distance between the observation planes. $\mathrm{K}$ is a number of these planes. Then the link between the wave field distributions at these planes takes the form

$$
u_{r}(x)=\left\{g_{r} * u_{0}\right\}(x), \quad g_{r}(x)=\left(-i z_{r} / \lambda \mathbf{r}^{2}\right) \cdot \exp (2 \pi i \lambda \mathbf{r}), \quad \mathbf{r}=\sqrt{z_{r}^{2}+\|x\|^{2}}
$$

Here $*$ denotes the convolution operator, $\lambda$ is the wavelength, and $g_{r}$ is the diffraction kernel for the distance $z_{r}$. In this case the intensity observations can be obtained using a moveable sensor ${ }^{18}$ (see Fig. 1a) placed at the measurement plane. The forward wave field propagation can be calculated in the Fourier transform domain as follows ${ }^{28-29}$

$$
U_{r}(\eta)=h_{r}(\eta) \cdot U_{0}(\eta), \quad h_{r}(\eta)=\exp \left(2 \pi i \cdot\left(z_{r} / \lambda\right) \cdot \sqrt{1-\lambda^{2}\|\eta\|^{2}}\right),\|\eta\|^{2} \leq \lambda^{2}
$$

with the spatial frequencies $\eta=\left(\eta_{1}, \eta_{2}\right)$. Since the transfer function $h_{r}$ describes only a phase modulation, the wave field propagation to the distance $z_{r}$ can be realized by a phase modulating spatial light modulator (SLM, see Fig. 1b) in the $4 \mathrm{f}$ optical system as it is originated in ${ }^{30}$. The sampled version of $h_{r}$ corresponding to $z_{r}$ is programmed on the SLM placed at the Fourier plane.

In our work we consider a discrete model (digital sensors, SLMs are 2D arrays of liquid crystal cells), assuming that the wave field at the object, Fourier and measurement (sensor) planes are pixel-wise invariant. Then, all continuous arguments are replaced by digital ones (e.g. $\left.x=\left(x_{1}, x_{2}\right) \rightarrow k=\left(k_{1}, k_{2}\right)\right)$ with the corresponding replacement of continuous distributions by their discrete counterparts (e.g. $\left.\mathrm{u}_{0}(x) \rightarrow \mathrm{u}_{0}[k]\right)$. Using the conventional vector-matrix notation the link between the planes can be rewritten as follows:

$$
\mathbf{u}_{r}=\mathbf{A}_{r} \cdot \mathbf{u}_{0}, r=1, \ldots \mathrm{K},
$$

where $\mathbf{A}_{r} \in \mathbb{C}^{\mathrm{n} \times \mathrm{n}}$ is a forward propagation operator; $\mathbf{u}_{0} \in \mathbb{C}^{n}$ and $\mathbf{u}_{r} \in \mathbb{C}^{n}$ are vectors, constructed by the column-wise concatenation of the 2D discrete wave field distributions (complex-valued $M_{x} \times M_{y}$ matrices) at the object and sensor planes, respectively. The length of the constructed vectors is $n=M_{x} \cdot M_{y}$. Various propagation operators, derived from the Rayleigh-Sommerfeld integral, can be used in the forward propagation modeling ${ }^{31,32}$. In this work we consider the object wave field reconstructions from the experimental data made according to the model presented in ${ }^{30}$. Thus, the wave field reconstructions are made by the conventional angular spectrum decomposition (ASD ${ }^{28}$ ). However, the computational performance is considered both for ASD and for the discrete diffraction transform in the spatial domain $\left(\mathrm{M}-\mathrm{DDT}{ }^{33}\right.$ ) as a propagation method for accurate pixel-to-pixel mapping from $\mathbf{u}_{0}$ to $\mathbf{u}_{r}$.

In the real optical setup the result of the wave field propagation is corrupted due to different distortions in the optical track as sensor noise, dust, focusing errors, etc. The intensity observation model is of the form 
(a)

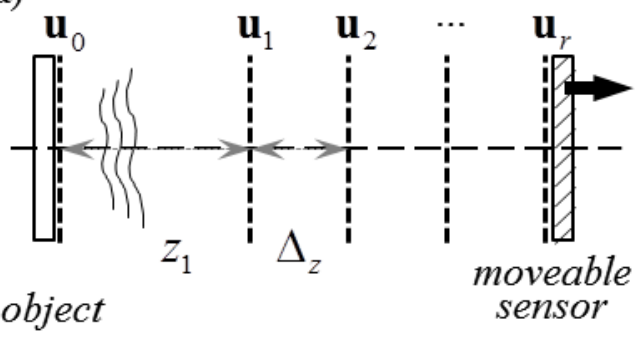

(b)

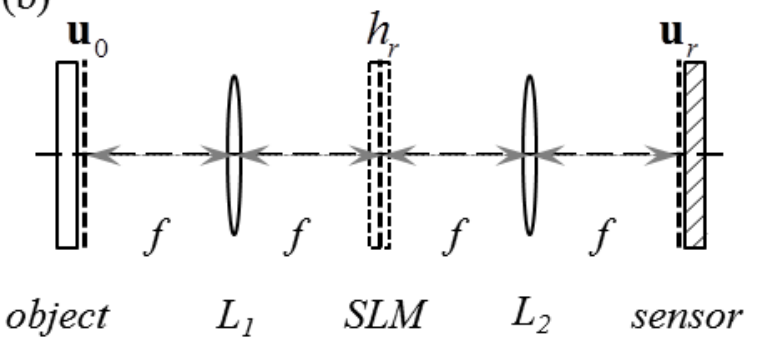

Figure 1. Optical models for phase retrieval: (a) free space propagation along the optical axis; (b) plane-to-plane propagation in a $4 \mathrm{f}$ system with filter operators $h_{r}$ programmed on the phase modulating SLM at the Fourier plane

${ }^{30}$. The lenses $L_{1}$ and $L_{2}$ with the focal distance $f$ provide mapping the object wave field to the sensor plane.

$$
\mathbf{o}_{r}=\left|\mathbf{u}_{r}\right|^{2}+\boldsymbol{\varepsilon}_{r}, r=1, \ldots \mathrm{K}
$$

with an additive error $\boldsymbol{\varepsilon}_{r}$. In this paper we consider the object reconstruction both by simulations with zero-mean Gaussian noise (for simplicity $\boldsymbol{\varepsilon}_{r}[k] \sim N\left(0, \sigma^{2}\right)$ with the same variation $\sigma^{2}$ for different planes as in ${ }^{1,24-27}$ ) and from experimental data with real disturbances in the optical track.

\subsection{Reconstruction formalization: constrained maximum likelihood and sparse regularization}

It is recognized that the forward wave field propagation cannot be compensated properly by the corresponding backward operator. The propagation operators, mapping an object wave field to a finite-size sensor at the observation plane, are illposed. Moreover, these operators cannot specify all disturbances of the optical track. In ${ }^{1}$ the object wave field estimate is found according to the variational maximum likelihood approach with the classical Tikhonov regularization. The object reconstruction is formulated as the following constrained optimization

$$
\mathbf{u}_{0}=\arg \min _{\mathbf{u}_{0}} \sum_{r=1}^{\mathrm{K}} \frac{1}{2 \sigma^{2}}\left\|\mathbf{o}_{r}-\left|\mathbf{u}_{r}\right|^{2}\right\|_{2}^{2}+\mu \cdot\left\|\mathbf{u}_{0}\right\|_{2}^{2} \quad \text { subject to } \mathbf{u}_{r}=\mathbf{A}_{r} \cdot \mathbf{u}_{0}, r=1, \ldots \mathrm{K},
$$

where the quadratic fidelity term appears due to our assumption that the observation noise is Gaussian, and $\mu>0$ is a regularization parameter included a prior to "stabilize" the solution in case of noise or ill-posed propagation operators $\mathbf{A}_{r}$. In ${ }^{22}$ it is shown that the adaptive regularization (realized via BM3D filter) results in a significant improvement in imaging. Thus, sparse modeling is decided to be included because of high performance and flexibility of this technique ${ }^{34}$ : it allows overcoming the loss of information due to the ill-posedness of forward propagation operators, wiping out different artifacts of the reconstructions, filtering noise, and therefore enhancing the resulting quality. The difference of this work concerns in separate sparse modeling for the object phase and amplitude via the powerful BM3D-frame filter, specified for denoising and other imaging problems ${ }^{24,35}$.

Following ${ }^{25-27}$ we use the object wave field in the form $\mathbf{u}_{0}=\mathbf{a}_{0} \circ \exp \left(j \cdot \boldsymbol{\varphi}_{0}\right)$, where "o" is the Hadamard product, and $\mathbf{a}_{0} \in \mathbb{R}^{n}$ and $\boldsymbol{\varphi}_{0} \in \mathbb{R}^{n}$ denotes the object amplitude and phase, respectively. According to the sparsity hypothesis we use an approximation of $\mathbf{a}_{0}$ and $\boldsymbol{\varphi}_{0}$ (separately) with small number of components of basis functions. Note that there is no prior information on the object modulation as in ${ }^{36}$. Sparse image approximation can be given in the synthesis or analysis form as follows:

$$
\begin{array}{lll}
\mathbf{a}_{0}=\boldsymbol{\Psi}_{a} \cdot \boldsymbol{\theta}_{a}, & \boldsymbol{\varphi}_{0}=\boldsymbol{\Psi}_{\varphi} \cdot \boldsymbol{\theta}_{\varphi} & \text { (synthesis) } \\
\boldsymbol{\theta}_{a}=\boldsymbol{\Phi}_{a} \cdot \mathbf{a}_{0}, & \boldsymbol{\theta}_{\varphi}=\boldsymbol{\Phi}_{\varphi} \cdot \boldsymbol{\varphi}_{0} & \text { (analysis) }
\end{array}
$$

Here $\boldsymbol{\Psi}$. and $\boldsymbol{\Phi}$. are the frame transform matrices, and the vector $\boldsymbol{\theta} . \in \mathbb{R}^{m}$ can be considered as a spectrum $(m \gg n)$ in a parametric data adaptive approximation (subindices $a$ and $\varphi$ are shown for the amplitude and phase, respectively). It is recognized that, in contrast to classical orthonormal bases $(m=n)$, overcomplete frame based modeling is a much more 
efficient for imaging ${ }^{34,37}$ and results in a better wave field reconstruction accuracy. The sparsity of approximation is characterized by either the $l_{0}$ norm $\|\boldsymbol{\theta}\|_{0}$ defined as a number of non-zero components of the vector $\boldsymbol{\theta}$ or the $l_{1}$ norm $\|\boldsymbol{\theta}\|_{1}$ as the sum of absolute values of items of the vector $\boldsymbol{\theta}$. Note that results obtained by $l_{0}$ or $l_{1}$ norms are shown to be closed to each other ${ }^{38}$. Taking into account the sparse modeling for the object amplitude and phase, the wave field reconstruction is performed by minimization of the criterion ${ }^{25-27}$

$$
\begin{array}{ll}
J=\sum_{r=1}^{\mathrm{K}} \frac{1}{2 \sigma^{2}}\left\|\mathbf{o}_{r}-\left|\mathbf{u}_{r}\right|^{2}\right\|_{2}^{2}+\tau_{a}\left\|\boldsymbol{\theta}_{a}\right\|_{p} & +\tau_{\varphi}\left\|\boldsymbol{\theta}_{\varphi}\right\|_{p} \text { subject to } \\
\mathbf{u}_{r}=\mathbf{A}_{r} \cdot \mathbf{u}_{0}, r=1, \ldots \mathrm{K}, & \text { (forward propagation) } \\
\boldsymbol{\theta}_{a}=\boldsymbol{\Phi}_{a} \cdot \mathbf{a}_{0}, \quad \boldsymbol{\theta}_{\varphi}=\boldsymbol{\Phi}_{\varphi} \cdot \boldsymbol{\varphi}_{0} & \text { (analysis) } \\
\mathbf{u}_{0}=\boldsymbol{\Psi}_{a} \cdot \boldsymbol{\theta}_{a} \circ \exp \left(j \cdot \boldsymbol{\Psi}_{\varphi} \cdot \boldsymbol{\theta}_{\varphi}\right) & \text { (synthesis) }
\end{array}
$$

where the regularization terms for the object amplitude and phase are taken using the $l_{o}$ or $l_{1}$ norms (in (7) $p=\{0,1\}$ ). The positive parameters $\tau_{a}$ and $\tau_{\varphi}$ define a balance between the fit to observations, smoothness of the wave field reconstruction and complexity of the model (cardinality of the spectra of the amplitude and phase). Instead of the singleobject minimization of $J$ we use a multi-objective optimization because of two reasons: simpler implementation (filtering and inverse procedure are decoupled) and the resulting better reconstruction quality (see e.g. ${ }^{24-27}$ ). Then the constrained minimization (7) can be rewritten in the unconstrained one with changing the constraints for sparse modeling by the quadratic penalties with positive weights. Therefore we introduce two criterion functions:

$$
\begin{gathered}
L_{1}=\sum_{r=1}^{\mathrm{K}} \frac{1}{\sigma^{2}}\left[\frac{1}{2}\left\|\mathbf{o}_{r}-\left|\mathbf{u}_{r}\right|^{2}\right\|_{2}^{2}+\frac{1}{\gamma}\left\|\mathbf{u}_{r}-\mathbf{A}_{r} \cdot \mathbf{u}_{0}\right\|_{2}^{2}+\frac{2}{\gamma} \operatorname{Re}\left\{\boldsymbol{\Lambda}_{r}^{H}\left(\mathbf{u}_{r}-\mathbf{A}_{r} \cdot \mathbf{u}_{0}\right)\right\}\right]+\frac{1}{\xi}\left\|\mathbf{u}_{0}-\mathbf{v}_{0}\right\|_{2}^{2} \\
L_{2}=\tau_{a}\left\|\boldsymbol{\theta}_{a}\right\|_{p}+\tau_{\varphi}\left\|\boldsymbol{\theta}_{\varphi}\right\|_{p}+\frac{1}{2 \chi_{a}}\left\|\boldsymbol{\theta}_{a}-\boldsymbol{\Phi}_{a} \cdot \mathbf{a}_{0}\right\|_{2}^{2}+\frac{1}{2 \chi_{\varphi}}\left\|\boldsymbol{\theta}_{\varphi}-\boldsymbol{\Phi}_{\varphi} \cdot \boldsymbol{\varphi}_{0}\right\|_{2}^{2}
\end{gathered}
$$

where $(\cdot)^{H}$ stands for the Hermitian conjugate, $\mathbf{v}_{0}=\boldsymbol{\Psi}_{a} \boldsymbol{\theta}_{a} \circ \exp \left(j \cdot \mathbf{\Psi}_{\varphi} \boldsymbol{\theta}_{\varphi}\right)$ is an approximation of the complex-valued object distribution $\mathbf{u}_{0}$. The synthesis and analysis constraints in (7) are replaced by the penalties with the corresponding positive parameters $\xi, \chi_{a}$ and $\chi_{\rho}$ in (8) and (9). The main difference from ${ }^{26,27}$ is the Lagrangian term in addition to the fidelity term in $L_{l}$. Thus, in (8) we have the augmented Lagrangian criterion ${ }^{39}$ with complex-valued variables: $\boldsymbol{\Lambda}_{r} \in \mathbb{C}^{\mathrm{n}}$ are the Lagrangian multipliers, the parameter $\gamma>0$ is equal for all planes. The usage both the linear and quadratic terms enables the stability of the saddle point of the augmented Lagrangian.

\section{DECOUPLED AUGMENTED LAGRANGIAN (D-AL) ALGORITHM}

It is recognized that the minimization of $L_{1}$ on $\mathbf{u}_{0}$ in general results in increasing of $L_{2}$ and vice versa, optimization of $L_{2}$ with respect to spectra $\boldsymbol{\theta}_{a}$ and $\boldsymbol{\theta}_{\varphi}$ increases $L_{l}$. This problem can be interpreted in terms of the game theory as a noncooperative interaction between the players. A compromise in this selfish behavior can be found in the fixed point $\left(\mathbf{u}_{0}{ }^{*}, \boldsymbol{\theta}_{a}{ }^{*}, \boldsymbol{\theta}_{\varphi}{ }^{*}\right)$ of the optimization called Nash equilibrium ${ }^{40,41}$. Then, the complex-valued object wave field distribution is reconstructed using decoupling of the inverse procedure and filtering of the object amplitude and phase (see also ${ }^{42}$ ).

$$
\left\{\begin{array}{c}
\left(\boldsymbol{\theta}_{a}^{*}, \boldsymbol{\theta}_{\varphi}^{*}\right)=\arg \min _{\boldsymbol{\theta}_{a}, \boldsymbol{\theta}_{\varphi}} L_{2}\left(\mathbf{a}_{0}^{*}, \boldsymbol{\varphi}_{0}^{*}, \boldsymbol{\theta}_{a}, \boldsymbol{\theta}_{\varphi}\right) \\
\mathbf{v}_{0}^{*}=\boldsymbol{\Psi}_{a} \cdot \boldsymbol{\theta}_{a}^{*} \circ \exp \left(j \cdot \boldsymbol{\Psi}_{\varphi} \cdot \boldsymbol{\theta}_{\varphi}^{*}\right) \\
\mathbf{u}_{0}=\arg \min _{\mathbf{u}_{0},\left\{\mathbf{u}_{r}\right\}} \max _{\left\{\boldsymbol{\Lambda}_{r}\right\}} L_{1}\left(\mathbf{u}_{0},\left\{\mathbf{u}_{r}\right\},\left\{\boldsymbol{\Lambda}_{r}\right\}, \mathbf{v}_{0}^{*}\right)
\end{array}\right.
$$

The algorithm solving the problem (10) has the following iterative form 


$$
\begin{gathered}
\left(\boldsymbol{\theta}_{a}^{t}, \boldsymbol{\theta}_{\varphi}^{t}\right)=\underset{\boldsymbol{\theta}_{a}, \boldsymbol{\theta}_{\varphi}}{\arg \min _{2}} L_{2}\left(\mathbf{a}_{0}^{t-1}, \boldsymbol{\varphi}_{0}^{t-1}, \boldsymbol{\theta}_{a}, \boldsymbol{\theta}_{\varphi}\right), \mathbf{v}_{0}^{t}=\boldsymbol{\Psi}_{a} \boldsymbol{\theta}_{a}^{t} \circ \exp \left(j \cdot \boldsymbol{\Psi}_{\varphi} \boldsymbol{\theta}_{\varphi}^{t}\right) \\
\mathbf{u}_{r}^{t}=\arg \min _{\mathbf{u}_{r}} L_{1}\left(\mathbf{u}_{0}^{t-1}, \mathbf{u}_{r}, \boldsymbol{\Lambda}_{r}^{t-1}\right), \quad r=1, \ldots \mathrm{K} \\
\boldsymbol{\Lambda}_{r}^{t}=\boldsymbol{\Lambda}_{r}^{t-1}+\alpha \cdot\left(\mathbf{u}_{r}^{t}-\mathbf{A}_{r} \cdot \mathbf{u}_{0}^{t-1}\right), \quad r=1, \ldots \mathrm{K} \\
\mathbf{u}_{0}^{t}=\arg \min _{\mathbf{u}_{0}} L_{1}\left(\mathbf{u}_{0},\left\{\mathbf{u}_{r}^{t}\right\},\left\{\boldsymbol{\Lambda}_{r}^{t-1}\right\}, \mathbf{v}_{0}^{t}\right), \mathbf{a}_{0}^{t}=\left|\mathbf{u}_{0}^{t}\right|, \boldsymbol{\varphi}_{0}^{t}=\arg \left\{\mathbf{u}_{0}^{t}\right\}
\end{gathered}
$$

Note that the minimization of $L_{2}$ is performed separately with respect to $\boldsymbol{\theta}_{a}$ and $\boldsymbol{\theta}_{\varphi}$. Depending on the chosen $l_{o}$ or $l_{1}$ norm in $L_{2}$ the so-called "soft" or "hard" thresholding is appeared for the amplitude or phase estimation. Indeed, we consider the optimization problem

$$
\frac{1}{2 \chi}\|\boldsymbol{\theta}-\mathbf{u}\|_{2}^{2}+\tau\|\boldsymbol{\theta}\|_{p} \rightarrow \min _{\boldsymbol{\theta}}
$$

for $p=\{0,1\}$. Note that according to the additive nature of the norms the problem can be solved independently for each component of $\boldsymbol{\theta}_{i}, i=1, \ldots m$. The solution of the optimization problem (12) is the following thresholding ${ }^{34}$ :

$$
\boldsymbol{\theta}=\mathfrak{I} h_{\tau \chi}(\mathbf{u})= \begin{cases}\operatorname{sigh}(\mathbf{u}) \circ(|\mathbf{u}|-\tau \chi)_{+} & \text {for } p=1 \\ \mathbf{u} \circ \mathbf{1}(|\mathbf{u}| \geq \sqrt{2 \tau \chi}) & \text { for } p=0\end{cases}
$$

where $(\cdot)_{+}$denotes the positive projection. The optimal complex-valued $\left\{\mathbf{u}_{r}{ }^{t}\right\}$ and $\mathbf{u}_{0}^{t}$ are calculated in accordance with the minimum conditions. Here the parameters $\sigma$ and $\gamma$ are the same for all $r$, thus only one parameter $\kappa=\sigma^{2} \gamma / \xi$ is used in the solution of the minimization of $L_{l}$ on $\mathbf{u}_{0}$. Minimization of $L_{l}$ on $\mathbf{u}_{r}$ is produced separately for every $r$ and $k$.

Similar to ${ }^{1}$ we are looking for the optimal fitting to the observations $\mathbf{o}_{r}$ by the nonlinear operator denoted as $\Gamma=\operatorname{argmin}_{\{\mathbf{u r}\}} L_{l}$ (see (A5) and (A6) in ${ }^{1}$ ). Such an optimization operation (the update of $\mathbf{u}_{r}{ }^{t}$ ) is denoted as "opt". In ${ }^{27}$ it is shown that for small amounts of noise the calculation of $\mathbf{u}_{r}{ }_{r}^{t}$ can be greatly simplified with a replacement of the calculated magnitude by the measured one as in the multi-plane phase-retrieval algorithms (e.g. ${ }^{22}$ ). This case is denoted as "MF".

Taking into account these two models of the recalculation of $\mathbf{u}_{r}{ }^{t}$, the solution of the multi-objective optimization (11) is calculated with the following iterative algorithm:

Initialization for $t=0: \mathbf{u}_{0}^{0}=\mathbf{a}_{0}^{0} \circ \exp \left(\mathrm{j} \varphi_{0}^{0}\right),\left\{\boldsymbol{\Lambda}_{r}^{0}\right\}$, transform matrices $\boldsymbol{\Psi}_{\mathrm{a}}, \boldsymbol{\Psi}_{\varphi}, \boldsymbol{\Phi}_{\mathrm{a}}$ and $\boldsymbol{\Phi}_{\varphi}$

Repeat for $t=1,2 \ldots$

$$
\begin{aligned}
& \boldsymbol{\theta}_{a}^{t}=\mathfrak{J} h_{\tau_{a} \chi_{a}}\left(\boldsymbol{\Phi}_{a} \cdot \mathbf{a}_{0}^{t-1}\right), \boldsymbol{\theta}_{\varphi}^{t}=\mathfrak{J} h_{\tau_{\varphi} \chi_{\varphi}}\left(\boldsymbol{\Phi}_{\varphi} \cdot \boldsymbol{\varphi}_{0}^{t-1}\right), \mathbf{v}_{0}^{t}=\boldsymbol{\Psi}_{a} \cdot \boldsymbol{\theta}_{a}^{t} \circ \exp \left(j \cdot \boldsymbol{\Psi}_{\varphi} \cdot \boldsymbol{\theta}_{\varphi}^{t}\right) \\
& \mathbf{u}_{r}^{t-1 / 2}=\mathbf{A}_{r} \cdot \mathbf{u}_{0}^{t-1}, \mathbf{u}_{r}^{t}[k]=\left\{\begin{array}{ll}
\sqrt{\mathbf{o}_{r}[k]} /\left|\mathbf{u}_{r}^{t-1 / 2}[k]\right| \cdot \mathbf{u}_{r}^{t-1 / 2}[k] & (\mathrm{MF}) \\
G\left(\mathbf{o}_{r}[k], \mathbf{u}_{r}^{t-1 / 2}[k], \boldsymbol{\Lambda}_{r}^{t-1}[k]\right) & (\mathrm{opt})
\end{array} \quad r=1, \ldots \mathrm{K}\right. \\
& \mathbf{B}_{r}=\left(\sum_{r=1}^{\mathrm{K}} \mathbf{A}_{r}^{H} \mathbf{A}_{r}+\kappa \mathbf{I}\right)^{-1} \cdot \mathbf{A}_{r}^{H}, \quad \mathbf{u}_{0}^{t}=\sum_{r=1}^{\mathrm{K}} \mathbf{B}_{r}\left(\mathbf{u}_{r}^{t}+\boldsymbol{\Lambda}_{r}^{t-1}\right)+\kappa \mathbf{v}_{0}^{t}, \mathbf{a}_{0}^{t}=\left|\mathbf{u}_{0}^{t}\right|, \boldsymbol{\varphi}_{0}^{t}=\arg \left\{\mathbf{u}_{0}^{t}\right\} \\
& \boldsymbol{\Lambda}_{r}^{t}=\boldsymbol{\Lambda}_{r}^{t-1}+\alpha \cdot\left(\mathbf{u}_{r}^{t}-\mathbf{u}_{r}^{t-1 / 2}\right), \quad r=1, \ldots \mathrm{K}
\end{aligned}
$$

End on $t$

The filtering operation (14) can be presented as follows:

1. Analysis. Highly correlated image blocks are distinguish and stacked together to form a 3D data array, which is decorrelated by an invertible 3D transform (calculation of the spectrum estimates $\boldsymbol{\theta}_{a}{ }^{t}$ and $\boldsymbol{\theta}_{\varphi}{ }^{t}$ ). 
2. Processing. 3D group spectra obtained from 3D data array are filtered by thresholding $\mathfrak{S} h$ (separately for the object amplitude and phase).

3. Synthesis. Filtered spectra are inverted providing estimates for each block in a group. These blocks are returned to their original positions, and then the final image estimate is aggregated by weighted averaging over all block-wise estimates (construction of the object estimate $\mathbf{v}_{0}{ }^{t}$ ).

The details related to the derivations of the main steps can be found in ${ }^{1,25-27}$. The augmented Lagrangian based phaseretrieval algorithm (14)-(17) is called decoupled augmented Lagrangian (D-AL), because the inverse procedure of the object wave field reconstruction (16) and the filtering operation of the reconstruction object amplitude and phase (what is different from the $\mathrm{AL}$ algorithm ${ }^{1}$ ) in the BM3D-frame domain (14) are decoupled.

\section{NUMERICAL EXPERIMENTS}

In our numerical experiments we consider the reconstruction of the amplitude-only object wave field distribution given as $\mathbf{u}_{0}=\mathbf{a}_{0}, \boldsymbol{\varphi}_{0}[\mathrm{k}]=0$, (for simulations $0.1 \leq \mathbf{a}_{0} \leq 1$ ), where $\mathbf{a}_{0}$ is a standard USAF 1951 test chart. In the discrete model the object and Fourier planes are assumed to be pixelated by square pixels of the size $\Delta_{0}=3.45 \mu \mathrm{m}$ and $\Delta_{\mathrm{s}}=8 \mu \mathrm{m}$, respectively, with $100 \%$ fill factor ${ }^{43}$. The wavelength $\lambda=532 \mathrm{~nm}$ corresponding to a green $\mathrm{Nd}$ :YAG laser. The presented reconstructions are given for $\mathrm{K}=5$ noisy observations with the fixed distance between the planes $\Delta_{z}=2 \mathrm{~mm}, z_{I}=20 \mathrm{~mm}$. The thresholding parameters for the amplitude $\tau_{a} \chi_{a}$ and phase $\tau_{\varphi} \chi_{\varphi}$ vary for different experiments. The regularization parameter of the AL algorithm is fixed and equal to $\mu=0.05$ (it is used because of noisy data). In D-AL we use fixed $\kappa=90 / 21$ in (16).

The initialization for $t=0$ concerns the object distribution (e.g. $\mathbf{u}_{0}^{0}[k]=1 / 2$ ), Lagrangian multipliers (e.g. $\left\{\boldsymbol{\Lambda}_{r}^{0}[k]\right\}=0$ ) and the BM3D-frames for the synthesis and analysis for both the object amplitude and phase. The updating step in (15) is taken small $\alpha=1 / 40$ for all $\mathrm{K}$ planes in the "opt" case. $\alpha=0$ for the "MF" case and the Lagrangian multipliers are not used $\left\{\boldsymbol{\Lambda}_{r}[\mathrm{k}]=0\right\}$ as in ${ }^{27}$.

The experimental results are shown for 100 iterations of the algorithm, including the initialization. The initial guess $\mathbf{u}_{0}{ }^{0}$ is calculated by AL during $T$ iterations. It is found that the duration $T$ of the calculation of an initial guess for $\mathbf{u}_{0}$ affects to the reconstruction quality: lack $\left(T<T_{\mathrm{p}}\right)$ or excess $\left(T>T_{\mathrm{p}}\right)$ of preprocessing may decrease the reconstruction accuracy (see Fig. 2).
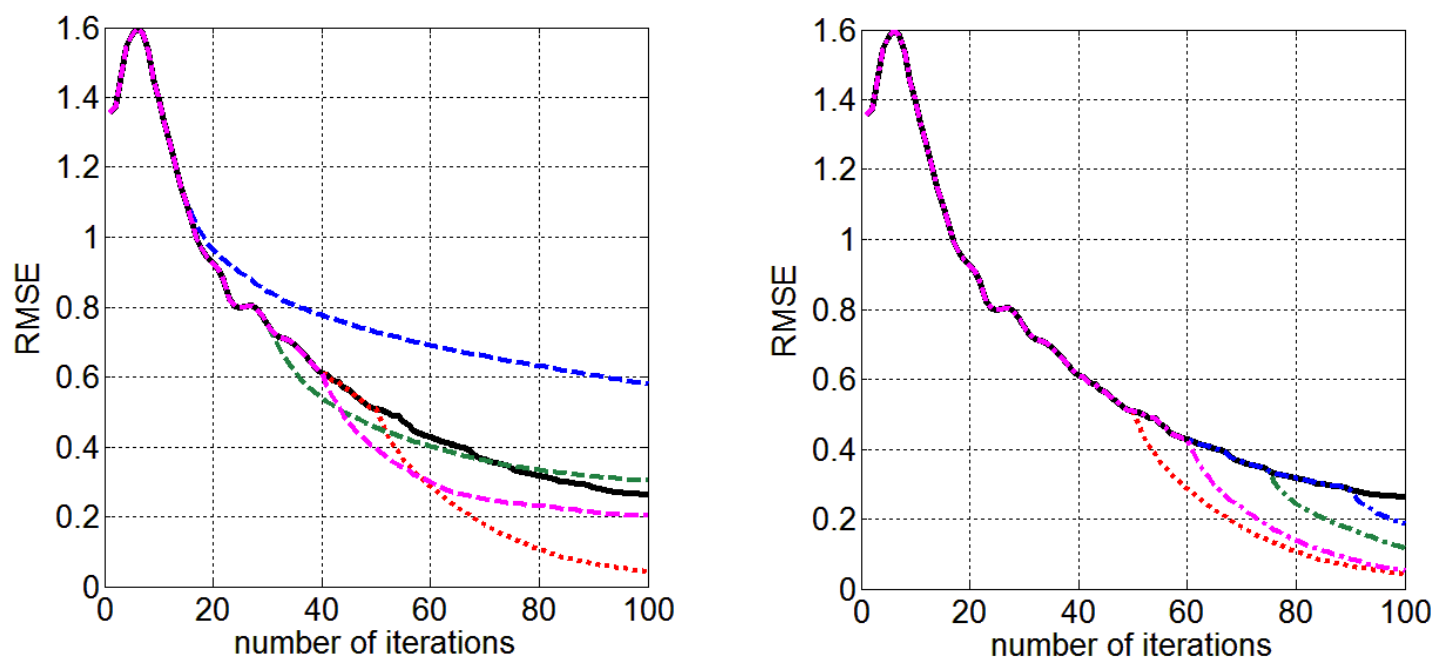

Figure 2. Convergence of the D-AL algorithm depending on the duration $T$ for the initial calculation of $\mathbf{u}_{0}^{0}$ by $\mathrm{AL}^{1}$ : (left figure, dashed curves) lack of preprocessing $\left(T<T_{\mathrm{p}}\right)$, (right figure, dash-dotted curves) excessive prereconstruction $\left(T>T_{\mathrm{p}}\right)$, (dotted curve) the proper choice $T_{\mathrm{p}}$. The solid curve corresponds to the object reconstruction by AL only. 


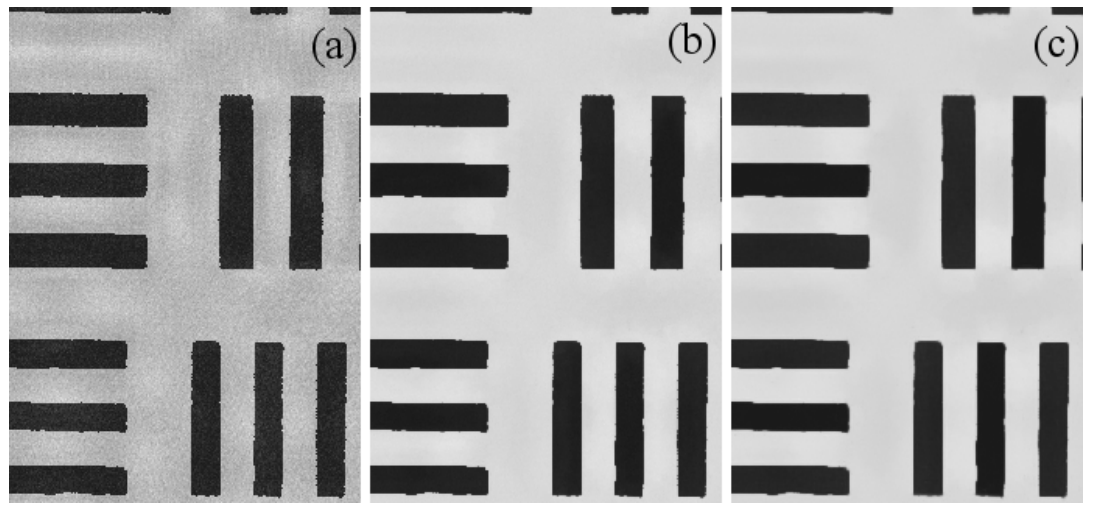

Figure 3. Fragments of the reconstructed amplitudes obtained by (a) AL ${ }^{1}, \operatorname{RMSE}\left(\mathbf{a}_{0}\right)=0.094$, (b) D-AL, "opt" case in (15), "soft" thresholding, $\tau_{a} \chi_{a}=0.05, \operatorname{RMSE}\left(\mathbf{a}_{0}\right)=0.039$ and (c) D-AL, "MF" case in (15), "hard" thresholding, $\tau_{a} \chi_{a}=0.1, \operatorname{RMSE}\left(\mathbf{a}_{0}\right)=0.043$. These results are presented for synthetic simulations, with zero-mean Gaussian noise in the observations, $\sigma=0.05, \mathrm{~K}=5$.

The proper duration of the object wave field preprocessing $\left(T_{\mathrm{p}}\right)$ is found here experimentally: we take 50 iterations for the object initialization by $\mathrm{AL}^{1}$ and 50 iterations for the D-AL algorithm (14)-(17).

\subsection{Numerical simulations}

In Fig. 3 we compare the reconstruction of the object amplitudes obtained from synthetic data by (from left to right) the original AL algorithm and two implementations of the D-AL algorithm realized according to the formulas, denoted as "opt" and "MF", respectively. The visual advantage of the D-AL algorithm is obvious. The corresponding cross-sections for these estimates are illustrated in Fig. 4. The wave field reconstruction accuracy is given in root-mean-square error (RMSE) values for the whole image of the size $M_{x} \times M_{y}=1024 \times 1024$.

It can be seen that the results of the "opt" and "MF" cases of the D-AL algorithm are closer to the true object shape, while the AL reconstruction is noisy and corrupted by clear diffraction artifacts. In order to suppress the artifacts and noise we apply BM3D-frame filtering with "soft" and "hard" thresholding with various parameters for the shrinkage. Firstly, it is found that the "opt" case, which is computationally more expensive, enables usually better reconstruction accuracy and a more contrast result. Here for the reconstruction with "soft" thresholding the "opt" case gives $\operatorname{RMSE}\left(\mathbf{a}_{0}\right)=0.039$, but $\operatorname{RMSE}\left(\mathbf{a}_{0}\right)=0.047$ for the "MF" case. Visually the difference concerns in smoothing of small details of the image.

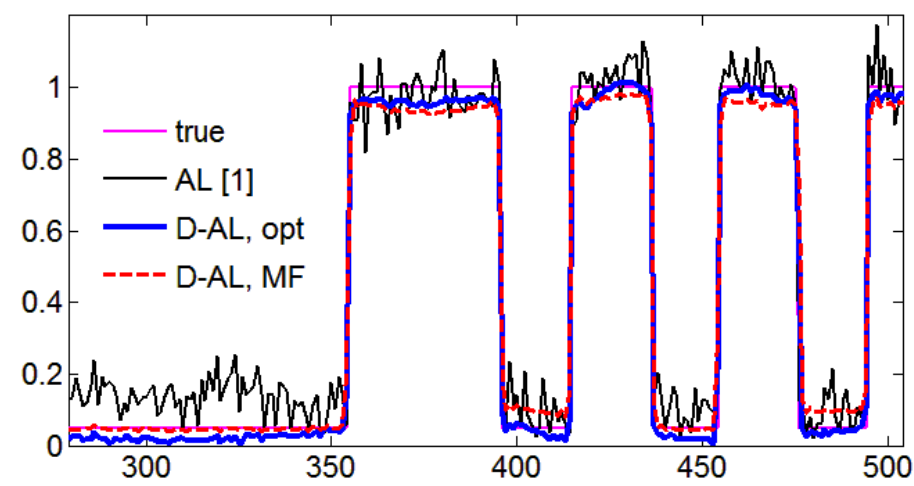

Figure 4. The cross-sections of the reconstructed amplitude for the tests presented in Fig. 3. 


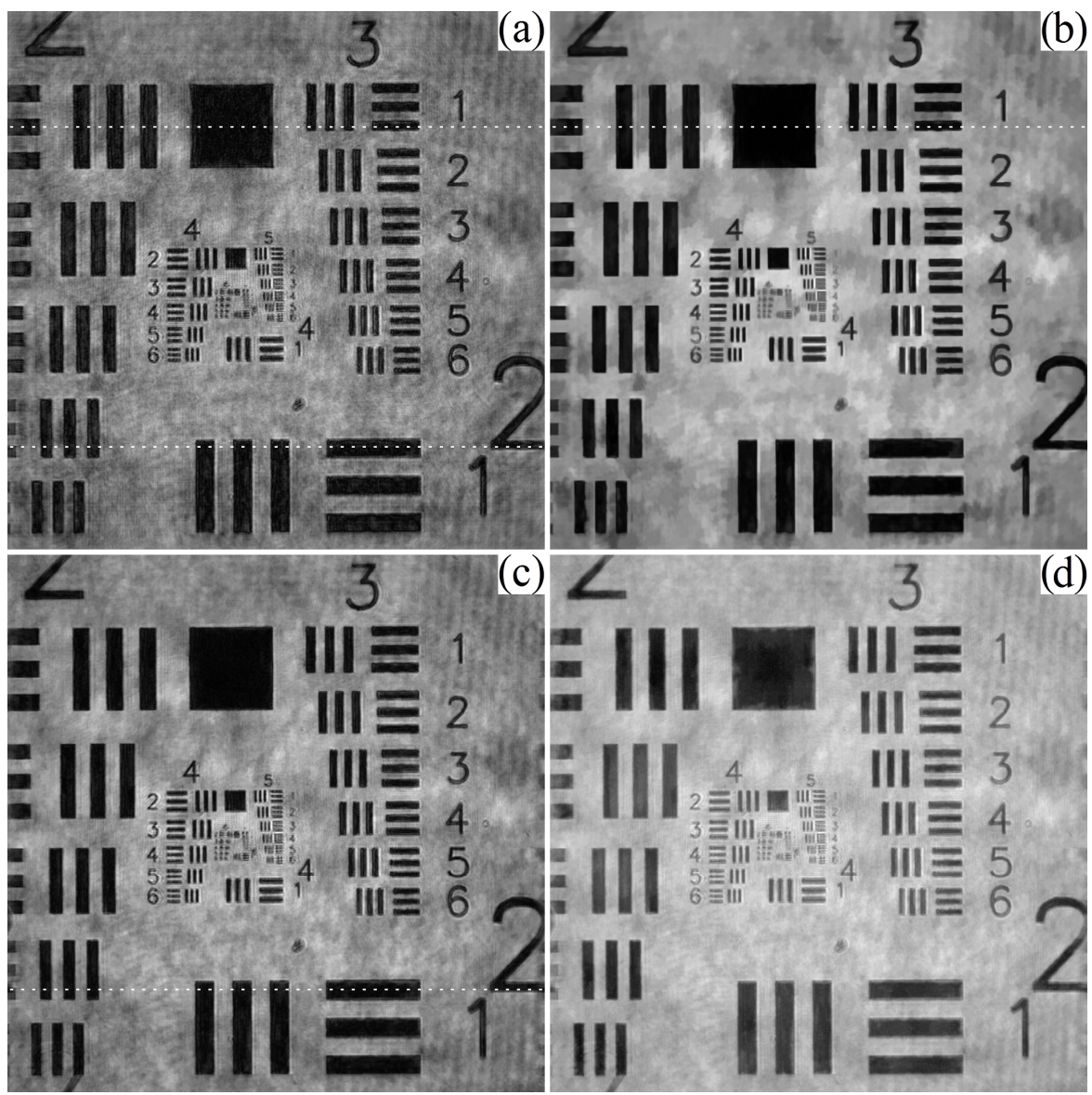

Figure 5. Reconstructions of the object amplitude from experimental data, obtained by (a) AL ${ }^{1}$, (b) D-AL "opt" with over-smoothing, $\tau_{a} \chi_{a}=0.2$, (c) D-AL "opt", $\tau_{a} \chi_{a}=0.05$ and (d) D-AL "opt" with over-smoothing, no filtering of the DC-term in the BM3D domain, $\tau_{a} \chi_{a}=0.9$.

Secondly, it is recognized that the "hard" thresholding is more efficient for noise suppression, however it may lead to blurring of reconstructing components (compare the borders of geometric elements in Fig. $3 \mathrm{~b}$ and $3 \mathrm{c}$ ). It is a challenge to find a balance for sharp imaging, especially in case of real experimental data with no true signal for testing: improper parameters result in oversmoothing or noisy reconstructions.

\subsection{Reconstructions from experimental data}

Further we present some examples of the amplitude estimates, obtained from the experimental data of the wave field propagation in the $4 \mathrm{f}$ system. These observations $\left\{\mathbf{o}_{r}\right\}$ are obtained in the Bremen Institute of Applied Beam Technology (BIAS, http://www.bias.de) according to the model originated in ${ }^{30,44}$. Here we reconstruct a part of the object distribution of the size $M_{x} \times M_{y}=1024 \times 1024$ for the related focal distance $f=53.125 \mathrm{~mm}^{31}$.

In Fig. 5 we compare the resulting imaging obtained by D-AL with various parameters of "soft" thresholding. It can be seen that the fluctuations (especially on the borders), which occur in the AL reconstruction (Fig. 5a) are partially suppressed by D-AL. Nevertheless, the reconstruction quality in case of large thresholding parameter (Fig. 5b) is poor because of oversmoothing. The reconstruction with a smaller thresholding parameter is sharper but noisy (see the corresponding cross-sections in Fig. 6b). It is found that a better imaging can be obtain by BM3D-frames with no filtering of the DC terms of the constructed 3D group and processing with respect to $-\mathbf{a}_{0}{ }^{t}+\max \left(\mathbf{a}_{0}{ }^{t}\right)$ instead of $\mathbf{a}_{0}{ }^{t}$. It enables a better suppression of "waves", but the image contrast is worse (see Fig. 5d). 


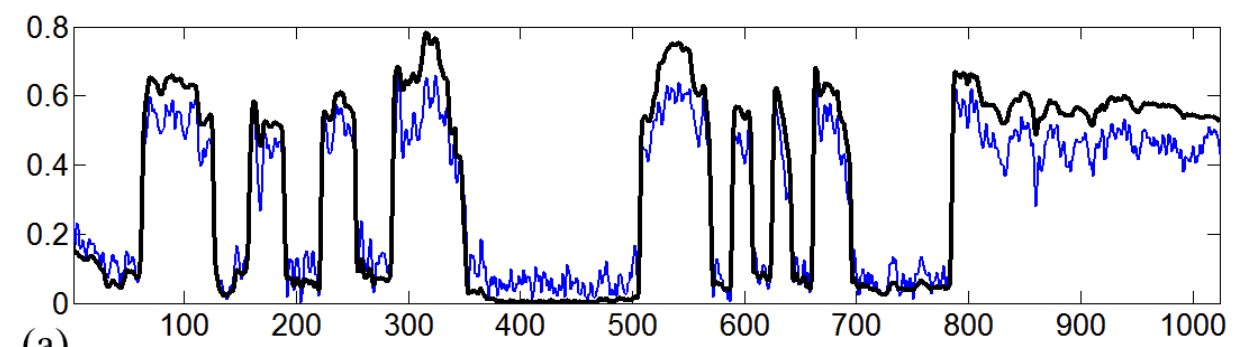

(a)

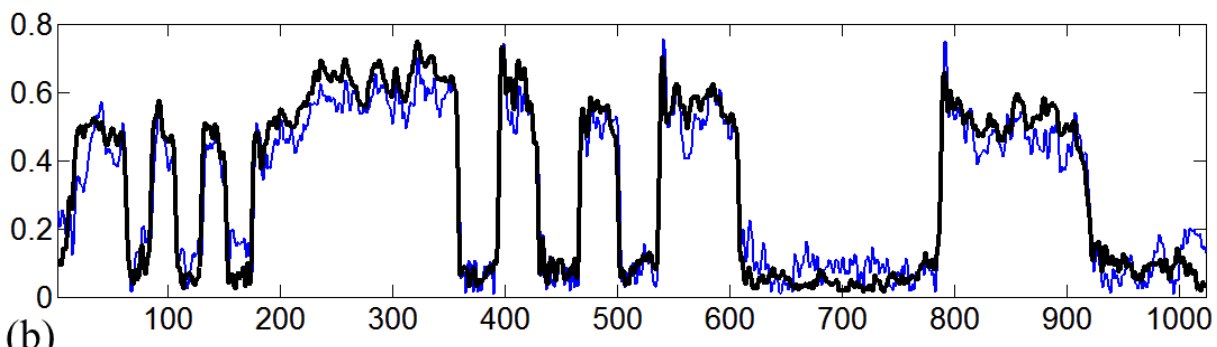

(b)

Figure 6. Cross-sections of the reconstructed amplitudes for the tests, presented in Fig. 5. The results are shown along the dotted lines shown in comparison of (a) Fig. 5a (here, thin curve) with Fig.5b (thick curve); (b) Fig. 5a (thin curve) with Fig. 5c (thick curve).

\subsection{Computational performance}

Here we present the comparison of computational performance for different implementations of the D-AL. It is clear that the operations (15)-(17) can be realized independently for any $r$-th plane and $k$-th component of the wave field distribution. In order to use the advantage of this parallel structure of the algorithm these operations are realized on a GPU. We demonstrate the results of simulations in MATLAB 7.13 (R2011b) using Nvidia GF460GTX with CUDA 4.1. The presented numbers are obtained by Monte Carlo simulations with averaging over 50 experiments. The computer used for experiments is Intel i5 2500 (4 physical cores) at 3.3 GHz; 8Gb RAM, Windows 7 SP1.

Since the BM3D-frame filtering is realized for CPU only, the overheads consists (mostly) of memory allocation and data transference between GPU and CPU: the amplitude $\mathbf{a}_{0}{ }^{t}$ and phase $\boldsymbol{\varphi}_{0}{ }^{t}$ estimates from GPU to CPU and back the filtered update of $\mathbf{v}_{0}{ }^{t}$. The profile of the presented speedup is calculated with respect to three time-consuming operations (15)(16) performed on GPU. Thus, the full procedure can be divided into four parts: the estimation of the wave fields at K sensor planes $\mathbf{u}_{r}^{t-1 / 2}$ (with the percentages of runtime $\mathrm{p}_{1}$ ), their update $\mathbf{u}_{r}{ }^{t}\left(\mathrm{p}_{2}\right)$ and the object reconstruction $\mathbf{u}_{0}^{t}\left(\mathrm{p}_{3}\right)$. Other operations including the update of $\left\{\boldsymbol{\Lambda}_{r}^{t}[k]\right\}$ (if $\alpha \neq 0$ ) and CPU/GPU transference are defined as overheads and takes the rest of runtime $\left(\mathrm{p}_{4}, \operatorname{sum}_{i}\left(\mathrm{p}_{i}\right)=1\right)$. In Table 1 the acceleration for these operations in average for $\mathrm{K}=\{3,5,8\}$ for square images of the size $M \times M, M=\{512,1024,2048\}$ are presented. Note that the "opt" and "MF" cases differ only in the update of $\mathbf{u}_{r}^{t}$

We consider the "opt" and "MF" cases for recalculation of the sensor wave field estimates $\mathbf{u}_{r}^{t}$ and compare two implementations of the wave field propagation realized via FFT, and in matrix form via DFT or using M-DDT ${ }^{33}$ (denoted as matrix in Table 1). The forward wave field propagation model (2) can be rewritten in terms of (3) via DFT as

$$
\begin{aligned}
& \mathbf{u}_{r}^{D F T}=\mathbf{W} \cdot\left(\mathbf{h}_{r} \circ\left(\mathbf{W} \cdot \mathbf{u}_{0}\right)\right), \mathbf{h}_{r}\left(\frac{\Delta_{s} v}{\lambda f}\right)=\int_{-\infty}^{\infty} \int_{-\infty}^{\infty} h_{r}(\eta) \delta\left(\eta-\frac{\Delta_{s} v}{\lambda f}\right) d \eta=\exp \left(\frac{2 \pi i z_{r}}{\lambda} \cdot \sqrt{1-\frac{\left\|v \Delta_{s}\right\|^{2}}{f^{2}}}\right), \\
& \mathbf{W}=\mathbf{W}_{x} \otimes \mathbf{W}_{y}, \mathbf{W}_{x}\left[v_{1}, k_{1}\right]=\exp \left(\frac{2 \pi \Delta_{0} k_{1} \Delta_{s} v_{1}}{i \lambda f}\right), \mathbf{W}_{y}\left[k_{2}, v_{2}\right]=\exp \left(\frac{2 \pi \Delta_{0} k_{2} \Delta_{s} v_{2}}{i \lambda f}\right),
\end{aligned}
$$

where $\otimes$ denotes the Kronecker product, $\mathbf{h}_{r}$ defines samples of $h_{r}, \delta$ is the 2D Dirac delta function and DFT expressed as Vandermonde matrices $\mathbf{W}_{x}$ and $\mathbf{W}_{y}$ for the spatial frequencies $v / \lambda f=\left(v_{1} / \lambda f, v_{2} / \lambda f\right)$. Note that here $\eta \in \mathbb{R}^{2}$ and $v \in \mathbb{Z}^{2}$. The 
accurate pixel-to-pixel model in terms of M-DDT (with "non-averaged" matrices $\mathbf{C}_{x}$ and $\mathbf{C}_{y}$ because the propagation is for the "in-focus" distance ${ }^{33}$ ) looks absolutely similar and gives a very close result

$$
\mathbf{u}_{r}^{M-D D T}=\mathbf{C}_{l} \mathbf{C} \cdot\left(\mathbf{h}_{r} \circ\left(\mathbf{C}_{l} \mathbf{C} \cdot \mathbf{u}_{0}\right)\right), \mathbf{C}=\mathbf{C}_{x} \otimes \mathbf{C}_{y}, \mathbf{C}_{l}=[l(:) \circ \mathbf{C}[:, 1], l(:) \circ \mathbf{C}[:, 2], \ldots]
$$

where $l(:)$ is the vector, constructed by columns concatenating of the $2 \mathrm{D}$ discrete distribution of the lens transmittance $l[k]=\exp \left(-\mathrm{i} \pi / \lambda f \cdot\|\mathrm{k}\|^{2}\right)$. Note that all these transform matrices $\mathbf{C}, \mathbf{C}_{l}$ and $\mathbf{W}$ are calculated only once. It is straightforward from (18) that the backward propagation for DFT based models is of the form ${ }^{22}$

$$
\mathbf{u}_{0}^{F F T}=\frac{1}{K} \sum_{r=1}^{K} \mathbf{W}^{-1} \cdot\left(\mathbf{h}_{r}^{-1} \circ\left(\mathbf{W}^{-1} \cdot \mathbf{u}_{r}\right)\right)
$$

and for M-DDT based model we have

$$
\mathbf{u}_{0}^{M-D D T}=\frac{1}{K} \mathbf{C}_{l}^{H} \mathbf{C}^{H} \cdot\left(\mathbf{h}_{r}^{-1} \circ\left(\mathbf{C}_{l}^{H} \mathbf{C}^{H} \cdot \mathbf{u}_{r}\right)\right), \mathbf{C}_{l}^{H}=\left[l(:)^{-1} \circ \mathbf{C}^{H}[:, 1], l(:)^{-1} \circ \mathbf{C}^{H}[:, 2], \ldots\right]
$$

Table 1. Computational accelerations $\mathrm{s}_{i}$ for different parts of the D-AL algorithm.

\begin{tabular}{|c|c|c|c|c|c|c|c|c|c|c|c|c|}
\hline $\begin{array}{c}\text { computational } \\
\text { algorithm }\end{array}$ & \multicolumn{3}{|c|}{ estimate $\mathbf{u}_{r}^{t-1 / 2}$} & \multicolumn{3}{|c|}{ update $\mathbf{u}_{r}{ }^{t}$} & \multicolumn{3}{c|}{ estimate $\mathbf{u}_{0}{ }^{t}$} & \multicolumn{3}{c|}{ overall speedup $S_{A}$} \\
\cline { 2 - 14 } $\begin{array}{c}\text { accelerating } \\
\text { operations (for } \\
M^{\times} M \text { image) }\end{array}$ & $\begin{array}{c}M= \\
512\end{array}$ & $\begin{array}{c}M= \\
1024\end{array}$ & $\begin{array}{c}M= \\
2048\end{array}$ & $\begin{array}{c}M= \\
512\end{array}$ & $\begin{array}{c}M= \\
1024\end{array}$ & $\begin{array}{c}M= \\
2048\end{array}$ & $\begin{array}{c}M= \\
512\end{array}$ & $\begin{array}{c}M= \\
1024\end{array}$ & $\begin{array}{c}M= \\
2048\end{array}$ & $\begin{array}{c}M= \\
512\end{array}$ & $M=$ & $M=$ \\
\hline FFT, "MF" & $\mathbf{4 . 5}$ & $\mathbf{7}$ & $\mathbf{4 . 4}$ & $\mathbf{9 . 3}$ & $\mathbf{1 1 . 4}$ & $\mathbf{9 . 5}$ & $\mathbf{5 . 1}$ & $\mathbf{9 . 1}$ & $\mathbf{9 . 4}$ & $\mathbf{5 . 9}$ & $\mathbf{8 . 9}$ & $\mathbf{4 . 3}$ \\
\hline matrix, "MF" & 2.8 & 1.8 & 1.1 & 9.4 & 11.3 & 9.4 & 2.8 & 1.9 & 1.3 & 3 & 1.9 & 1.3 \\
\hline FFT, "opt" & 4.5 & 7 & 4.4 & 1.3 & 2 & 2.8 & 5.1 & 9.1 & 9.4 & 1.4 & 2 & 3 \\
\hline matrix, "opt" & 2.8 & 1.8 & 1.1 & 1.2 & 2.1 & 2.9 & 2.8 & 1.9 & 1.3 & 1.7 & 1.9 & 1.4 \\
\hline
\end{tabular}

Taking into account the overheads, the overall speedup $\mathrm{S}_{A}$ of the algorithm is found according to Amdahl's law 45,46

$$
S_{A}=1 /\left(\sum_{i} p_{i} / s_{i}\right)
$$

where $\mathrm{s}_{i}$ is the acceleration (speedup) of the program part $\mathrm{p}_{i}$. It is well-known that $\mathrm{S}_{A}$ is usually substantially smaller than the improvement found for individual parts because of different value of $\mathrm{p}_{i}$ relative to the whole program and additional overheads. In our case the realization via FFT with simple replacement of the magnitude ("MF" case) demonstrates the best performance. High rate of the overall speedup $\mathrm{S}_{A}$ is due to the approximately uniform distribution of the computational consumption: for the presented operation $\mathrm{p}_{l: 3}=\{29,28,38\}(\%)$ in average. The worst case is for the FFT realization with the optimization of the sensor plane wave fields ("opt" case), because the accelerated operations take a minor part: the computational consumption is $\mathrm{p}_{1: 3}=\{7,81,9\}(\%)$. The greater the overheads the smaller the benefit from the parallelization. In our case it is mostly because of limited RAM of GPU, and the performance decreases significantly: see the results for $M_{x} \times M_{y}=2048 \times 2048$.

\section{CONCLUSION}

In this paper the augmented Lagrangian based phase-retrieval algorithm with powerful BM3D-frame filtering is analyzed for various propagation scenarios. We consider the reconstruction quality of the algorithm and computational performance by mean of implementation using a graphic processing unit in the $4 \mathrm{f}$ system and in the free space propagation (depending on the used transform matrices). The developed phase-retrieval algorithm is treated as a further 
development of $\mathrm{AL}{ }^{1}$ with sparse approximation both for amplitude and phase objects. The D-AL algorithm demonstrates a significant improvement of the reconstruction accuracy and enhanced imaging comparing with AL both for noisy synthetic and experimental observation data. It is shown that the parallel processing with a commodity GPU results in essential acceleration of the wave field recalculation: even in MATLAB we have a good acceleration for the whole algorithm up to 9 times. It is found that for worse CPUs (e.g. with one or 2 cores) the contrast is more significant: for Intel Core 2 Duo E8400 at $3 \mathrm{GHz} \mathrm{S}_{A}$ the speedup for FFT, "MF" is approximately 20 times. The presented GPU based realization of D-AL can be considered as an easy solution for processing of large images, and it is a good motivation for the further more specific implementations.

\section{REFERENCES}

[1] Migukin, A., Katkovnik, V. and Astola, J., "Wave field reconstruction from multiple plane intensity-only data: Augmented Lagrangian algorithm", J. Opt. Soc. Am. A, 28, 993-1002 (2011).

[2] Gerchberg, R. W. and Saxton, W. O., "A practical algorithm for the determination of phase from image and diffraction plane pictures," Optik 35, 237-246 (1972).

[3] Gonsalves, R. A., "Phase retrieval from modulus data," J. Opt. Soc. Am. 66, 961-964 (1976).

[4] Yang, G., Dong, B., Gu, B., Zhuang, J. and Ersoy, O., "Gerchberg-Saxton and Yang-Gu algorithms for phase retrieval in a nonunitary transform system: a comparison," Appl. Opt. 33(2), 209-218 (1994).

[5] Fienup, J. R., "Reconstruction of an object from the modulus of its Fourier transform," Opt. Lett. 3, 27-29 (1978).

[6] Misell, D. L., "A method for the solution of the phase problem in electron microscopy," J. Phys. D 6, L6-L9 (1973).

[7] Zalevsky, Z., Mendlovic, D. and Dorsch, R. "Gerchberg-Saxton algorithm applied in the fractional Fourier or the Fresnel domain," Opt. Lett. 21, 842-844 (1996).

[8] Gureyev, T. E., "Composite techniques for phase retrieval in the Fresnel region," Opt. Commun. 220, 49-58 (2003).

[9] Fienup, J. R., "Iterative method applied to image reconstruction and to computer generated holograms," Opt. Eng. 19, 297-305 (1980).

[10] Katkovnik, V., Astola, J. and Egiazarian, K., "Wavefield Reconstruction and Design as Discrete Inverse Problems," 3DTV08, 265-268 (2008).

[11]Fienup, J. R., "Phase retrieval algorithms: A comparison," Appl. Opt. 21(15), 2758-2769 (1982).

[12] Crimmins, T. R. and Fienup, J. R., "Uniqueness of phase retrieval for functions with sufficiently disconnected support," J. Opt. Soc. Am. 73, 218-221 (1983).

[13] Seldin, J. H. and Fienup, J. R., "Numerical investigation of the uniqueness of phase retrieval," J. Opt. Soc. Am. A 7, 412-427 (1990).

[14] Allen L. and Oxley, M. P., "Phase retrieval from series of images obtained by defocus variation," Opt. Commun. 199, 65-75 (2001).

[15] Ivanov, V. Yu., Sivokon, V. P. and Vorontsov, M. A., "Phase retrieval from a set of intensity measurements: theory and experiment," J. Opt. Soc. Am. A 9(9), 1515-1524 (1992).

[16]Zhang, Y., Pedrini, G., Osten, W. and Tiziani, H., "Whole optical wave field reconstruction from double or multi in-line holograms by phase retrieval algorithm," Opt. Express 11(24), 3234-3241 (2003).

[17] Sinclair, G., Leach, J., Jordan, P., Gibson, G., Yao, E., Laczik, Z., Padgett, M. and Courtial, J., "Interactive application in holographic optical tweezers of a multi-plane Gerchberg-Saxton algorithm for three-dimensional light shaping," Opt. Express 12, 1665-1670 (2004).

[18] Almoro, P., Pedrini, G. and Osten, W., "Complete wavefront reconstruction using sequential intensity measurements of a volume speckle field," Appl. Opt. 45(34), 8596-8605 (2006).

[19] Teague, M., "Deterministic phase retrieval: a Green's function solution," J. Opt. Soc. Am. A 73(11), 1434-1441 (1983).

[20] Soto, M. and Acosta, E., "Improved phase imaging from intensity measurements in multiple planes," Appl. Opt. 46(33), 7978-7981 (2007). 
[21] Paganin, D., Barty, A., McMahon, P. J. and Nugent, K., "Quantitative phase-amplitude microscopy. III. The effects of noise," J. Microsc. 214(1), 51-61 (2004).

[22] Migukin, A., Katkovnik, V. and Astola, J., "Multiple plane phase retrieval based on inverse regularized imaging and discrete diffraction transform," AIP Conf. Proc.1236, ICAPMMOI (2010).

[23] Gazit, S., Szameit, A., Eldar, Y. C. and Segev, M., "Super-resolution and reconstruction of sparse subwavelength images," Opt. Express 17, 23920-23946 (2009).

[24] Katkovnik, V., Danielyan, A. and Egiazarian, K., "Decoupled inverse and denoising for image deblurring: variational BM3D-frame technique," in Proc. of IEEE International Conf. on Image Proc. (ICIP, 2011).

[25] Migukin, A., Katkovnik, V. and Astola, J., "Advanced phase retrieval: maximum likelihood technique with sparse regularization of phase and amplitude," arXiv:1108.3251v1.

[26] Katkovnik, V. and Astola, J., "High-accuracy wave field reconstruction: decoupled inverse imaging with sparse modeling of phase and amplitude," J. Opt. Soc. Am. A 29, 44-54 (2012).

[27] Katkovnik, V. and Astola, J., "Phase retrieval via spatial light modulator phase modulation in 4f optical setup: numerical inverse imaging with sparse regularization for phase and amplitude," J. Opt. Soc. Am. A 29, 105-116 (2012).

[28] Goodman, J., W., [Introduction to Fourier Optics], 2nd ed. McGraw-Hill (1996).

[29] Sherman, G.C., "Application of the convolution theorem to Rayleigh's integral formulas," J. Opt. Soc. Am. 57, 546-547 (1967).

[30] Falldorf, C., Agour, M., Kopylow, C. and Bergmann, R. B., "Phase retrieval by means of a spatial light modulator in the Fourier domain of an imaging system," Appl. Opt. 49, 1826-1830 (2010).

[31] Kreis, Th., [Handbook of Holographic Interferometry, Optical and Digital Methods], Wiley-VCH (2005).

[32] Shen F. and Wang, A., "Fast-Fourier-transform based numerical integration method for the RayleighSommerfeld diffraction formula," Appl. Opt. 45, 1102-1110 (2006).

[33] Katkovnik, V., Migukin, A. and Astola, J., "Backward discrete wave field propagation modeling as an inverse problem: toward perfect reconstruction of wave field distributions," Appl. Opt. 48, 3407-3423 (2009).

[34]Elad, M., [Sparse and Redundant Representations: From Theory to Applications in Signal and Image Processing], Springer (2010).

[35] Chatterjee, P. and Milanfar, P., "Is denoising dead?" IEEE Trans. Image Process. 19, 895-911 (2010).

[36] Migukin, A., Katkovnik, V. and Astola, J., "Optimal phase retrieval from multiple observations with Gaussian noise: augmented Lagrangian algorithm for phase objects," Proc. SPIE 8082, 80820L (2011).

[37] Han, D., Kornelson, K., Larson, D. and Weber, E., [Frames for Undergraduates], Student Mathematical Library, AMS, (2007).

[38] Donoho, D. L., "Compressed sensing," IEEE Trans. Inf. Theory 52, 1289-1306 (2006).

[39] Hestenes, M. R., "Multiplier and gradient methods," J. Optim. Theory Appl. 4, 303 - 320 (1969).

[40] Facchinei, F. and Kanzow, C., "Generalized Nash equilibrium problems," 4OR: A Quarterly Journal of Operations Research 5, 173-210 (2007).

[41] Kulkarni, A.A. and Shanbhag, U.V., "New insights on generalized Nash games with shared constraints: Constrained and variational equilibria," IEEE Conf. on Decision and Control, 151-156 (2009).

[42]Danielyan, A., Katkovnik, V. and Egiazarian, K., "BM3D frames and variational image deblurring", IEEE Trans. Image Process., preprint (2011).

[43] Arizon, V., Carreon, E. and Testorf, M., "Implementation of Fourier array illuminators using pixelated SLM: efficiency limitations," Optics comm. 16, 207-213 (1999).

[44] Agour, M., Falldorf, C., Kopylow, C. and Bergmann R. B., "Automated compensation of misalignment in phase retrieval based on a spatial light modulator," Appl. Opt. 50, 4779-4787 (2011).

[45] Amdahl, G.M., "Validity of the single-processor approach to achieving large-scale computing capabilities," AFIPS Conf. Proc., 483-485 (1967).

[46] Gustafson, J. L., "Reevaluating Amdahl's Law," Communications of the ACM 31, 532-533 (1988). 